\title{
A Double-Layer Model for Describing the Effect of Solvation and Adsorption of Ions on Electrode Surfaces in Batteries
}

\author{
J. Lück ${ }^{\text {a,b.c }}$ and A. Latz ${ }^{\mathrm{a}, \mathrm{b}, \mathrm{c}}$ \\ ${ }^{a}$ Institute of Engineering Thermodynamics, German Aerospace Center (DLR), 70569 \\ Stuttgart, Germany \\ ${ }^{\mathrm{b}}$ Helmholtz Institute Ulm for Electrochemical Energy Storage (HIU), 89081 Ulm, \\ Germany \\ ${ }^{\mathrm{c}}$ Institute of Electrochemistry, University of Ulm, $89081 \mathrm{Ulm}$, Germany
}

\begin{abstract}
We developed a consistent model to describe the effect of the electrochemical double-layer on the exchange reaction occurring at the interface of an electrode and a concentrated electrolyte. This model includes electrosorption, i.e. an accumulation of surface charges, and the screening of these charges within the electrochemical double-layer. We determine charges of the doublelayer only from fundamental thermodynamic and electrostatic considerations, without assuming any specific structural properties of the electrolyte-electrode interface. This mathematical description is integrated in a thermodynamic consistent reactiontransport model for lithium-ion batteries (6).
\end{abstract}

\section{Introduction}

Without a doubt the development of highly efficient electrochemical energy storage devices can be called the missing link that is needed for making renewable energy sources ready for daily use. To achieve this substantial aim a deep understanding of what is happening inside electrochemical cells is required to give research the opportunity to find improved systems. Whereas a lot of research is done regarding bulk properties of electrolytes and insertion materials, like structural changes or transport processes, little is known about the processes at the electrolyte-electrode interface, like adsorption and desolvation or the influence of the electrochemical double-layer. Detailed models for the transport of ions in the electrode and in the electrolyte have been developed, but the exchange reaction occurring at the interface between electronic and ionic conductor is mostly treated with a simple Butler-Volmer ansatz. Following this approach, surface processes like electrosorption and the electrochemical double-layer are not taken into account.

Contrary to this theoretical work, Bruce et al. $(1,2)$ showed in 1992 with their impedance measurements that electrosorption is indeed a crucial process for insertion materials and that electrointercalation must be understood as at least a two-step process.

According to the growth mechanism for metal desposition, they proposed their so-called adatom model for electrointercalation, which is shown in Fig. 1. There the fully solvated lithium ion in bulk electrolyte partially loses its solvation shell before adsorbing on the surface. Then it diffuses to a surface site, where it can intercalate into the electrode. 


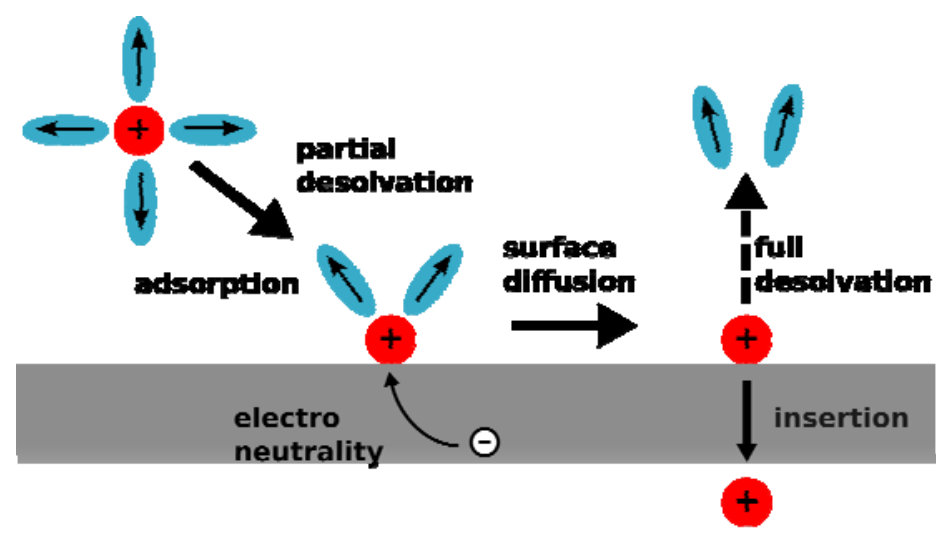

Figure 1. Schematic representation of the lithium intercalation reaction at the electrolyteelectrode interface: In the adatom model the fully solvated lithium ion in bulk electrolyte loses parts of its solvation shell, while adsorbing on the electrode surface. Then the adion diffuses to a surface site, where it can intercalate into the electrode. There it loses the remaining solvent and gets incorporated in the lattice structure of the active material.

After this, the adion loses the remaining solvent and gets incorporated in the lattice of the electrode. Thus, surface diffusion is fast and never rate-limiting, so that the intercalation reaction consists of an adsorption step with partial desolvation, followed by the actual insertion step. This model was successfully applied to different insertion materials as well (3-5).

Following these experimental indications we put our focus on modeling the exchange reaction as a two-step process to add electrosorption and the electrochemical double-layer to the theoretical description of batteries. A theory based only on thermodynamic derivations allows us to obtain a comprehensive understanding of the reaction mechanism taking place at the electrochemical interface and provides a scientific base for predictive models. We believe that this is a relevant process of understanding both intercalation and conversion batteries.

\section{Modeling approach}

Starting from the observation of $(1,2)$, it is reasonable to deduce that the exchange reaction at the electrochemical interface of an intercalation battery should be at least described as a two-step process. Considering lithium-ion batteries during discharge, first an adsorption step occurs

$$
\mathrm{Li}^{+}[\mathrm{aq}]+\mathrm{S}+(1-\delta) \mathrm{e}^{-} \rightarrow \mathrm{LiS}^{+\delta}
$$

where a lithium-ion $\mathrm{Li}^{+}$is adsorbed at a surface site $\mathrm{S}$, while possibly changing its state of charge to $+\delta$. Then the actual insertion step can take place

$$
\mathrm{LiS}^{+\delta} \rightarrow \mathrm{Li}^{+}[\mathrm{s}]+\mathrm{S}+(1-\delta) \mathrm{e}^{-}
$$

as a transition of the lithium between an adsorbed state and an inserted state in the electrode structure. This assumes that the ionic nature of $\mathrm{Li}^{+}$, i.e. the state of charge is preserved within the complete intercalation reaction from electrolyte to solid phase. For graphitic materials this assumption is supported by $(7,8)$. 


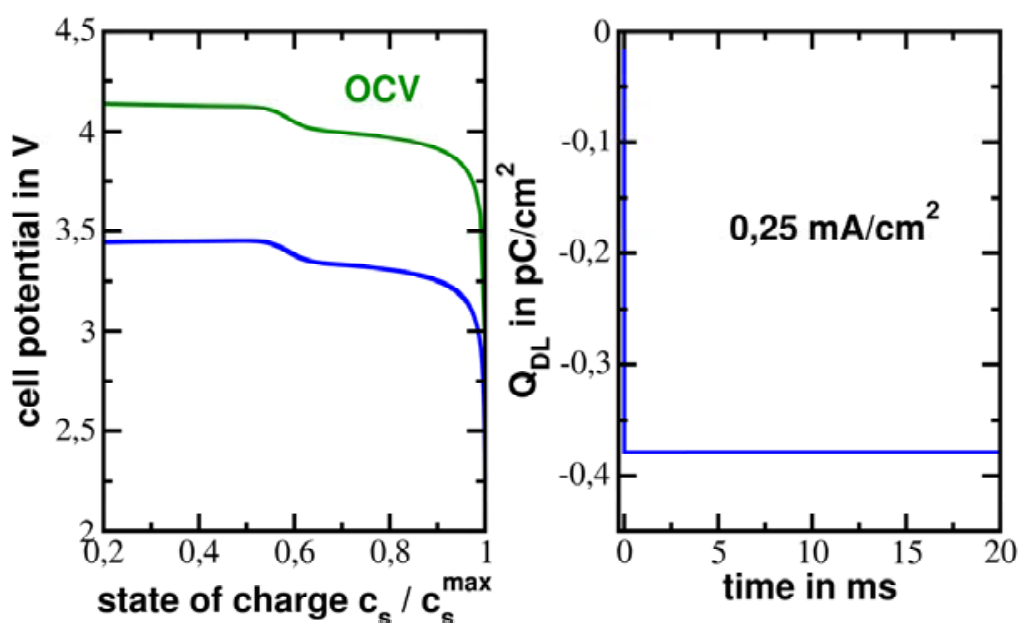

Figure 2. Simulation results for a half cell calculation without transport limitations at a constant current discharge: (left) State of charge reaches 1 because we neglected transport effects to focus on the influence of the interface. (right) The charge stored in the doublelayer attains a fixed value after a parameter-dependent time interval of approximately 1 $\mu \mathrm{s}$.

In the framework of standard theories of reaction-transport models for lithium-ion batteries we introduce an intermediate phase between a concentrated electrolyte and some active material. This intermediate phase is given by the adsorption of charged species on the electrode surface. To describe these surface charges we make use of the electric field occurring at the electrochemical interface. Compensating charges in the electrode and in the electrolyte are calculated analogously, where a compensation ratio between solid and electrolyte is brought into use. In equilibrium a compensation ratio can be determined from balancing forces at the interface.

The adsorption phase is coupled to the transport processes in electrolyte and active material by respective interface conditions for the ionic flux and the electric current. These conditions are derived from the assumption that all $\mathrm{Li}^{+}$ions are transferred between the particular phases. Lithium ions are not stored in one phase and there is no flux of negative charges through the double-layer. Further, we assume that the doublelayer can be charged and is so not always electroneutral. The exchange currents between the chemical phases are given by a Butler-Volmer approach.

\section{Results \& Conclusion}

Our model is implemented in a thermodynamic consistent reaction-transport model for lithium-ion batteries (6). Figure 2 shows simulation results for a half cell calculation while galvanostatic discharge. Here we focus on the influence of the electrode-electrolyte interface and therefore neglect transport effects. This yields a charging of the doublelayer in the beginning of discharge, a fixed value during discharge and electroneutrality in equilibrium, which demonstrates a consistent calculation of charge accumulation at the interface. In this setting the constant charge in the double-layer in quasi-equilibrium is reached after approximately $1 \mu \mathrm{s}$. This time interval depends on the chosen parameters. 
The surface coverage attains a reasonable value, so that electrosorption and the effect of the electrochemical double-layer on the exchange mechanism is modeled accurately.

The mathematical description of the accumulation of surface charges and the compensating charges in the electrode and the electrolyte is obtained only by fundamental thermodynamic and electrostatic considerations. No structural properties of the double-layer, as e.g. its thickness, are used. Therefore the model allows some insight into the chemical and physical processes at the electrode-electrolyte interface and their influence on the operation of a battery. Furthermore the description of an electrolyteelectrode interface is applicable to both conversion and intercalation systems. A detailed derivation of the discussed model and a precise analysis of the results will be presented in future work.

\section{References}

1. P.G. Bruce and M.Y. Saidi, J. Electroanal. Chem., 322, 92-105 (1992).

2. P.G. Bruce and M.Y. Saidi, Solid State Ionics, 51, 187-190 (1992).

3. S. Kobayashi et al., J. Phys. Chem. B, 109, 13322-13326 (2005).

4. M. Nakayama et al., J. Phys. Chem. C, 118, 27245-27251 (2014).

5. M. Nakayama et al., J. Phys. Chem. B, 107, 10603-10607 (2003).

6. A. Latz et al., J. Power Sources, 196, 3296-3302 (2011).

7. K. Xu, J. Electrochem. Soc., 157 (3), A162-A167 (2007).

8. J.R. Dahn et al., Science, 270, 590-593 (1995). 